\title{
The effects of ulinastatin in multiple organ failure: a retrospective observational study in a single center ICU
}

\author{
M Uchida ${ }^{*}$, T Abe $^{2}$, K Ono $^{1}$ \\ From ESICM LIVES 2015 \\ Berlin, Germany. 3-7 October 2015
}

\section{Introduction}

Ulinastatin, an urinary protease inhibitor, might reduce mortality of patients with systemic inflammatory response syndrome such as ARDS [1] and sepsis [2].

\section{Objectives}

Our aim was to evaluate the effectiveness of ulinastatin on 28-day mortality among patients with multiple organ failure.

\section{Methods}

We conducted a retrospective observational study of patients who were diagnosed multiple organ failure within 24 hours from admission in a general ICU of a tertiary care hospital in Japan. The study duration was from January 2009 to December 2012. The primary outcome was 28-day all cause mortality. The intervention was received ulinastatin for three days and more. Patients were also stratified by category of diseases as subgroup analysis.

\section{Results}

A total of 212 patients with multiple organ failure met inclusion criteria during this study period. At baseline, mean age was $66.2 \pm 14.7$ years old, male was 146/212 (69\%), mean APACHE II score was $24.7 \pm 7.9,206 / 212(97 \%)$ patients received mechanical ventilation, and 205/212 (96\%) patients were on vasopressor. Overall, 79/212 (37\%) patients received ulinastatin. 28-day all cause mortality was $43 / 212$ (20\%). There were no significant differences between ulinasatin group and control group in age, gender, APACHE II score. Ulinastatin group had higher prevalence of sepsis

'Dokkyo Medical University, Department of Emergency and Critical Care Medicine, Shimotsuga-gun Mibu-machi, Japan

Full list of author information is available at the end of the article
(35/79 (44.3\%) vs. $29 / 133$ (21.8\%), $P=0.001)$. They were more likely to receive corticosteroid, vasopressor, renal replacement therapy, and veno-arterial extra-corporeal membranous oxygenation (VA-ECMO). The mortality was not significantly different between the ulinastatin group and control group (20/79 (25.3\%) vs. 23/133 (17.3\%), $P=0.163)$. In logistic regression after adjusting for APACHE II score, there was not significant difference in the mortality (OR = 1.59; $95 \% \mathrm{CI}, 0.79-3.21$ ). The result was similar after adjusting for APACHE II score, sepsis, respiratory disorder, therapeutic interventions including corticosteroid, vasopressor, renal replacement therapy, and VA-ECMO. (OR $=1.29$; 95\% $\mathrm{CI}, 0.56$ - 2.99). In sepsis patients, ulinastatin did not reduce the mortality, either. $(\mathrm{OR}=1.92 ; 95 \% \mathrm{CI}, 0.52-7.13)$.

\section{Conclusions}

Ulinastatin was not associated with survival in patients with multiple organ failure.

\section{Authors' details \\ 'Dokkyo Medical University, Department of Emergency and Critical Care Medicine, Shimotsuga-gun Mibu-machi, Japan. ${ }^{2}$ Tsukuba Medical Center Hospital, Department of Emergency and Critical Care Medicine, Tukuba-shi, Japan.}

Published: 1 October 2015

\section{References}

1. Leng $Y-X$, et al: Ulinastatin for acute lung injury and acute respiratory distress syndrome: A systematic review and meta-analysis. World I Crit care Med 2014, 3:34-41.

2. Karnad DR, et al: Intravenous administration of ulinastatin (human urinary trypsin inhibitor) in severe sepsis: a multicenter randomized controlled study. Intensive Care Med 2014, 40:830-8.

doi:10.1186/2197-425X-3-S1-A878

Cite this article as: Uchida et al:: The effects of ulinastatin in multiple organ failure: a retrospective observational study in a single center ICU. Intensive Care Medicine Experimental 2015 3(Suppl 1):A878.

\section{SpringerOpen ${ }^{\circ}$}

(0) 2015 Uchida et al.; This is an Open Access article distributed under the terms of the Creative Commons Attribution License (http:// creativecommons.org/licenses/by/4.0), which permits unrestricted use, distribution, and reproduction in any medium, provided the original work is properly cited. 